\title{
CARACTERÍSTICA DEMOGRÁFICA E DA TERAPIA INTRAVENOSA PERIFÉRICA EM CRIANÇAS/ ADOLESCENTES COM CÂNCER ASSOCIADO A OCORRÊNCIA DE EXTRAVASAMENTO
}

\author{
Larissa Freitas Cerqueira ${ }^{1}$; Luciano Marques dos Santos ${ }^{2}$; Cleonara Sousa Gomes e \\ Silva $^{3}$ e Hérica Martins Barreto Carvalho ${ }^{4}$ \\ 1. Bolsista PROBIC/UEFS, Graduando em Enfermagem, Universidade Estadual de Feira de Santana, e-mail: \\ cerqueirafreitaslarissa@gmail.com \\ 2. Orientador, Departamento de Saúde, Universidade Estadual de Feira de Santana, e-mail: lucmarxenfo@ yahoo.com.br \\ 3. Participante do projeto "Segurança do paciente pediátrico e sua família: estudo de tecnologias e eventos adversos \\ associado a terapia intravascular periférica”, Departamento de Saúde, Universidade Estadual de Feira de Santana, e-mail: \\ cleosilvauefs@gmail.com \\ 4. Participante do projeto "Segurança do paciente pediátrico e sua família: estudo de tecnologias e eventos adversos \\ associado a terapia intravascular periférica”, Departamento de Saúde, Universidade Estadual de Feira de Santana, e-mail: \\ martins.herica@hotmail.com
}

PALAVRAS CHAVES: Criança hospitalizada; Extravasamento de materiais terapêuticos e diagnósticos; Segurança do paciente

\section{INTRODUÇÃO}

Segundo o Instituto Nacional de Câncer (2015) o câncer infanto juvenil acomete crianças e adolescentes entre 0 e 19 anos, é um conjunto de doenças que apresentam características próprias. É considerada uma doença rara, correspondendo entre $1 \%$ e $3 \%$ de todos os tumores malignos na maioria das populações.

Para o tratamento do câncer existem quatro modalidades terapêuticas: a excisão cirúrgica, a irradiação, a quimioterapia e a terapia biológica, sendo estas combinadas ou utilizadas isoladamente (DINIZ et al., 2008).

A quimioterapia intravenosa configura-se, no conjunto das modalidades utilizadas no tratamento do câncer, como estratégia que pode potencializar a cura do câncer. Entretanto, a sua utilização poderá comprometer a segurança da criança que dela faz utilização, tendo em vista o potencial risco para a ocorrência de eventos adversos, com destaque para o extravasamento (SCHNEIDER; PEDROLO, 2011).

O extravasamento é a infiltração de fármacos ou soluções com propriedades vesicantes para tecidos adjacentes, sendo caracterizado pela capacidade de produzir vesículas no local da lesão (INS-BRASIL, 2013).

Sendo uma das complicações mais graves em paciente que realizam a quimioterapia, devido ao potencial nocivo de tais drogas, pois muitos deles são irritantes, podem formar bolhas ou necrose em tecidos que se infiltram. O dano produzido irá depender da droga extravasada, do seu volume e sua concentração (VILLARÍN; BELDA, 2004). Entretanto, não há na literatura tanto nacional quanto internacional informações claras sobre os fatores que contribui para a ocorrência de extravasamento em crianças com câncer.

Portanto, este trabalho terá como objetivo verificar condições demográficas e da terapia intravenosa (TIV) prévia de crianças e adolescentes com cateteres vasculares periféricos para a terapia infusional associados à ocorrência de extravasamento em crianças com câncer em um hospital pediátrico de Feira de Santana, Bahia.

\section{METODOLOGIA}


Trata-se de um estudo transversal realizado na unidade de oncologia de um hospital pediátrico em Feira de Santana-Bahia, no período de abril de 2015 a dezembro de 2016. Os participantes foram crianças/adolescentes que tinham entre 29 dias e 16 anos. Foram utilizados como critérios de inclusão, ter idade de 29 dias e 16 anos incompletos, ter a necessidade de terapia intravenosa através de cateter venoso periférico, ter um quadro clinico estável e apresenta-se consciente.

Como critérios de não inclusão: as crianças e adolescentes em situação de isolamento, e que utilizaram o cateter intravenoso periférico e cateter venoso central concomitantemente. Os participantes da amostra foram acompanhados desde a realização da cateterização intravenosa periférica até o momento da retirada do cateter. Estes participantes foram divididos em dois grupos: os que retiram o cateter por conta da finalização da terapia intravenosa e os que apresentaram extravasamento.

Os dados destes participantes foram coletados por meio dos prontuários dos pacientes. As informações pertinentes ao estudo foram transcritas para o formulário de coleta de dados. Os dados foram digitados e analisados no programa Statistical Package for Social Sciences (SPSS), versão 22.0. e Open Epi Versão 3.01.

Para descrição das variáveis de cada categoria foram utilizados dados relativos à frequência absoluta e relativa. Nas variáveis numéricas foram analisados as médias e o desvio-padrão. A avaliação das associações entre as variáveis de exposição e desfecho foi realizada através do cálculo da razão de prevalência (RP) com intervalo de confiança de $95 \%$.

Foram utilizados os testes de hipótese qui-quadrado de Pearson e Teste Exato de Fisher, para comparar os fatores que predispõem à ocorrência de extravasamento em as crianças e adolescentes com câncer adotando como nível de significância os valores igual ou inferior a $5 \%$.

A pesquisa foi aprovada pelo Comitê de Ética em Pesquisa da Universidade Estadual de Feira de Santana, através do parecer de número 841.612

\section{RESULTADOS}

Com relaçao às características relacionadas à criança/adolescente, observou-se associação estatística entre os grupos com extravasamento e sem extravasamento para a condição nutricional $(p=0,021)$ e tempo de hospitalização $(p=0,003)$. Crianças/adolescentes desnutridas apresentaram prevalência três vezes maior do que as eutróficas para a ocorrência de extravasamento, assim como crianças/adolescentes que ficaram internadas por mais de oito dias apresentaram prevalência seis vezes maior do que as que ficaram internadas por até sete dias.

Tabela 1: Associação entre as variáveis relacionadas às crianças/adolescentes e a ocorrência de extravasamento em uma unidade pediátrica no interior da Bahia, Feira de Santana, no período de 2015 e 2016.

\begin{tabular}{|c|c|c|c|c|c|}
\hline \multirow[b]{2}{*}{ Variáveis } & \multicolumn{5}{|c|}{ Extravasamento } \\
\hline & $\begin{array}{c}\operatorname{Sim}(\mathbf{n}=26) \\
\quad \mathbf{N}(\%)\end{array}$ & $\begin{array}{c}\text { Não }(\mathbf{n}=273) \\
\quad \mathbf{N}(\%)\end{array}$ & $\mathbf{R P}$ & IC & P-valor \\
\hline \multicolumn{6}{|l|}{ Idade } \\
\hline $\begin{array}{l}\geq \text { a } 7 \text { anos }(84 \text { meses ou } \\
\text { mais) }\end{array}$ & $\begin{array}{l}12(9,9) \\
14(7,9)\end{array}$ & $\begin{array}{l}109(90,1) \\
164(92,1)\end{array}$ & 1,26 & $0,604-2,631$ & $0,536^{*}$ \\
\hline \multicolumn{6}{|l|}{ Até 6 anos (até 83 meses) } \\
\hline \multicolumn{6}{|l|}{ Sexo } \\
\hline Feminino & $15(9,9)$ & $136(90,1)$ & 1,34 & $0,635-2,813$ & $0,443 *$ \\
\hline Masculino & $11(7,4)$ & $137(92,6)$ & & & \\
\hline \multicolumn{6}{|l|}{ Raça/cor } \\
\hline Brancas & $9(9,7)$ & $84(90,3)$ & 1,17 & $0,543-2,533$ & $0,686^{*}$ \\
\hline Negras & $17(8,3)$ & $189(91,7)$ & & & \\
\hline
\end{tabular}




\begin{tabular}{|c|c|c|c|c|c|}
\hline \multicolumn{6}{|c|}{ Condição nutricional a } \\
\hline Desnutrida & $6(23,1)$ & $20(76,9)$ & 3,07 & 1,354-6,959 & $0,021 *$ \\
\hline Eutrófica & $20(7,5)$ & $246(92,5)$ & & & \\
\hline \multicolumn{6}{|c|}{$\begin{array}{l}\text { Tempo de hospitalização } \\
\text { (dias) }\end{array}$} \\
\hline$\geq$ a 8 dias & $4(50,0)$ & $4(50,0)$ & 6,61 & 2,969-14,734 & $\mathbf{0 , 0 0 3} * *$ \\
\hline Até 7 dias & $22(7,6)$ & $269(92,4)$ & & & \\
\hline
\end{tabular}

FONTE: Coleta de dados, Feira de Santana - Bahia, 2015/2016.

a Foram, excluídas dessa variável 7 crianças classificadas como obesas, pois as mesmas não apresentaram extravasamento. *Qui-quadrado de Person

**Teste exato de Fisher

Conforme a Tabela 1, o extravasamento foi mais prevalente em crianças com idade de até 6 anos (7,9\%), do sexo feminino $(9,9 \%)$ e que se autodeclararam como negras $(8,3 \%)$.

De acordo com a Tabela 2, que descreve as variáveis relacionada as características da TIV prévia observou-se associação estatística, entre os grupos, para histórico de dificuldade da inserção da CIP $(\mathrm{p}=0,001)$, TIV periférica prolongada $(\mathrm{p}=0,001)$ e antecedentes de complicação $(\mathrm{p}=0,009)$. Crianças/adolescentes com histórico de dificuldade de inserção da CIP apresentaram prevalência três vezes maior do que as que não tem dificuldade, para a ocorrência de extravasamento.

Da mesma forma, crianças/adolescentes com TIV periférica prolongada apresentaram prevalência seis vezes maior do que as que não utilizaram TIV periférica prolongada, para a ocorrência de extravasamento. Crianças/adolescentes com antecedentes de complicação apresentaram prevalência cinco vezes maior das que não tem histórico de complicação.

Tabela 2: Associação entre as variáveis relacionadas à TIV prévia e a ocorrência de extravasamento em uma unidade pediátrica no interior da Bahia, Feira de Santana, no período de 2015 e 2016.

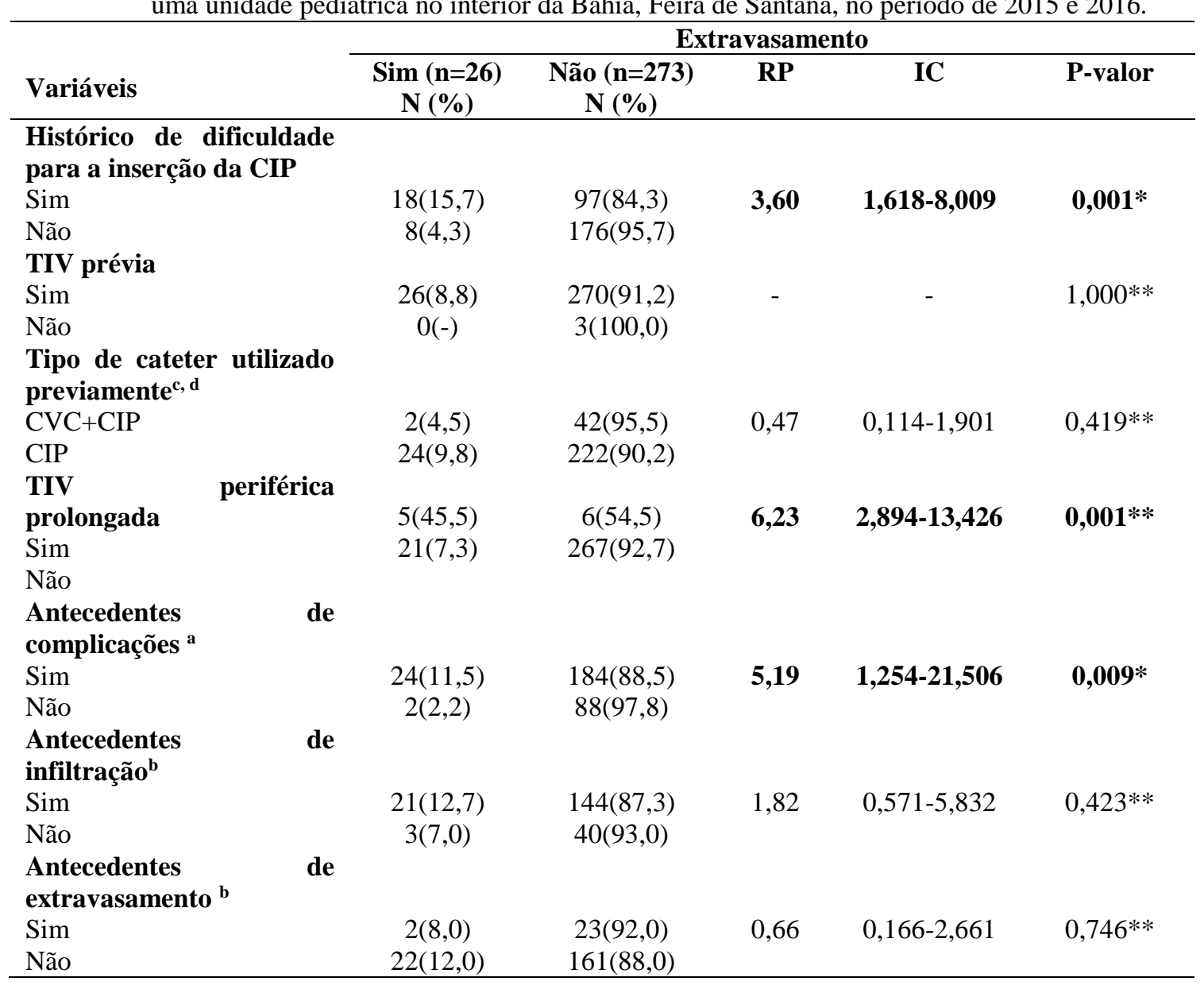


FONTE: Coleta de dados, Feira de Santana - Bahia, 2015/2016.

a Em 1 CIP não foi possível visualizar essa variável.

b Em 91 CIP não foi possível visualizar essa variável.

c Forem excluídas dessa variável 2 CIP classificadas com CIP+CVC+PICC e 4 com CIP+PICC pois não apresentaram extravasamento.

d Em 3 CIP não foi possível visualizar essa variável.

*Qui-quadrado de Person

**Teste exato de Fisher

Conforme a Tabela 2 nota-se uma maior prevalência de extravasamento nas crianças/adolescentes que utilizaram TIV prévia $(8,8 \%)$, sendo cateter intravenoso periférico o mais utilizado previamente $(9,8 \%)$ que tiveram antecedentes de infiltração $(12,7 \%)$ e não apresentaram antecedentes de extravasamento $(12 \%)$.

\section{CONCLUSÃO}

A ocorrência de extravasamento está estatisticamente associada às características demográficas quanto à condição nutricional, tempo de hospitalização e da TIV prévia, quanto à histórico de dificuldade de inserção da CIP, TIV periférica prolongada e antecedentes de complicação. Sendo imprescindível que profissionais de saúde que estabelecem a terapia infusional associem as características demográficas e da TIV prévia de crianças e adolescentes com câncer à ocorrência de extravasamento.

Os dados obtidos contribuirão para conhecer as características da demográficas das crianças/adolescentes e da TIV prévia que predispõe ao risco de extravasamento em cateteres vasculares inseridos perifericamente em crianças com câncer. Auxiliará a prática clínica, permitindo a prevenção desta complicação, contribuindo com a segurança do paciente, evitando o extravasamento de agentes quimioterápicos e com isso a diminuição do tempo de hospitalização da criança com câncer.

\section{REFERÊNCIA}

DINIZ, R. P. E. et al. Efeitos adversos identificados em local de infusão intravenosa periférica por drogas quimioterápicas. Cienc.Enferm., v. 14, n. 2, p. 55-64. 2008. Disponível em: <http://www.scielo.cl/pdf/cienf/v14n2/art08.pdf >. Acesso em: 20 jan. 2016

INSTITUTO NACIONAL DE CÂNCER. Estimativa 2016: incidência de câncer no Brasil. Rio de Janeiro, 2015. Disponível em:

<http://www.inca.gov.br/bvscontrolecancer/publicacoes/edicao/Estimativa_2016.pdf >. Acesso em: 20 jan. 2016.

INFUSION NURSES SOCIETY BRASIL. Diretrizes práticas para a terapia infusional. São Paulo, 2013.

SCHNEIDER, F.; PEDROLO, E. Extravasamento de drogas antineoplásicas: avaliação do conhecimento da equipe de enfermagem. Rev. Min. Enfer., v. 15, n. 4, p. 522-29, out./dez. 2011. Disponível em: < http://www.reme.org.br/artigo/detalhes/66 >. Acesso em: 15 jan. 2016

VILLARÍN, A. J. L.; BELDA, J. N. Prevención y tratamiento de lasextravasaciones de quimioterapia intravenosa. Enfermería Clínica, v.14, n. 2, p. 122-26, 2004. Disponível em: < http://apps.elsevier.es/watermark/ctl_servlet?_f=10\&pident_articulo=13061313\&pident_usua rio $=0 \&$ pcontactid $=\&$ pident_revista $=35 \&$ ty $=162 \&$ accion $=$ L\&origen $=$ zonadelectura $\& w e b=w w$ w.elsevier.es\&lan $=$ es\&fichero $=35$ v14n02a13061313pdf001.pdf $>$. Acesso em: 12 fev. 2016 